\title{
Development of Quantitative Usability Evaluation Method
}

\author{
Shin'ichi Fukuzumi ${ }^{1}$, Teruya Ikegami ${ }^{1}$, and Hidehiko Okada ${ }^{2}$ \\ ${ }^{1}$ NEC Corporation, Common Platform Software Research Laboratories, \\ 7-1, Shiba 5-chome, Minato-ku, Tokyo 108-8001, Japan \\ \{s-fukuzumi@aj,t-ikegami@ct\}.jp.nec.com \\ ${ }^{2}$ Kyoto Sangyo University, Kamigamo Motoyama, Kita-ku, Kyoto 603-8555, Japan \\ hidehiko@cc.kyoto-su.ac.jp
}

\begin{abstract}
A variety of evaluation methods are practiced in order to make more appealing and improve the usability of computer systems. The authors have developed a quantitative usability evaluation method that uses a checklist that outlines an evaluation procedure and clarifies judging standards. This paper describes this quantitative usability evaluation method that is not influenced by an evaluator's subjective impression. Moreover, such clear and precise definitions makes checklist-based evaluations more repeatable (thus more reliable) and less affected by differences among evaluators. The effectiveness of our checklist has been evaluated by the experiments with novice and experienced evaluators. This article reports the method and results of the experiments.
\end{abstract}

Keywords: Usability, evaluation, checklist.

\section{Introduction}

A usability evaluation method using a checklist [1], which is typical in usability evaluations [2], can be applied to the later stages of a development process [3]. However, obtaining justifiable evaluation results is difficult because they depend on the evaluators' skill, experience, and subjectivity. To solve this problem, we have developed a usability checklist to minimize deviation of evaluation results for realizing usability quantification [4-5]. In this paper, we introduce this checklist, validate it, and apply it to system operation products.

\section{Development of a Checklist}

\subsection{Problem of Usability Quantification and Approach for Its Solution}

Exclusion of an evaluator's skill. Generally, a score of goodness of fit for an evaluation target is obtained by using a checklist evaluation method. However, when evaluating over five stages, the results will blur due to the subjectivity of evaluators [6]. Also, sometimes an evaluator cannot understand the meaning of the contents of the item and misinterprets it. For example, the names of UI parts, such as "a list box" and "a pull-down menu", vary in meaning even among expert evaluators. Because of this, wrong items are chosen as evaluation targets. 
The authors made sure to judge each item as "has a problem", "No problems" or "irrelevant" for a clarifying procedure, an evaluation target, and a gauge. Moreover, to stop evaluators' different intelligibilities and interpretations causing results to blur, samples in a checklist and a collection of terminology definitions were also prepared.

Visualization of the effect to the user. An evaluation axis often consists of an element directly connected with design and development, such as a layout, and a button because it is assumed that a specialist in UI design and a developer generally use a checklist, and the effect for the user is hard to determine. To measure the degree to which the effect of each item of the checklist satisfies the user, it is important to weight the qualities of each item.

The authors weighted the items using the analytic hierarchy process (AHP) method [7], and evaluation results were decided on the basis of four qualities: "efficiency", "ease to learn", "errors", and "ease to memorize".

\subsection{Maintenance of a Checklist}

Selection of the item. The authors referred to the various standards and guidelines, made a rough draft of the checklist, selected items, improved them through verifying them, and then evaluated them. Moreover the AHP method of weighting the items was contemplated, and the chapter construction of the item was decided so that no part of the layer became too deep (table 1).

Table 1. Chapter of the checklist

\begin{tabular}{|c|l|c|}
\hline & Section name & The number of items \\
\hline 1 & Consistency of indication/ operation & 17 \\
\hline 2 & Legibility of information & 22 \\
\hline 3 & Presentation of the present state & 18 \\
\hline 4 & Conformability to the user/ environment & 19 \\
\hline 5 & Conformability to the work & \\
\hline
\end{tabular}

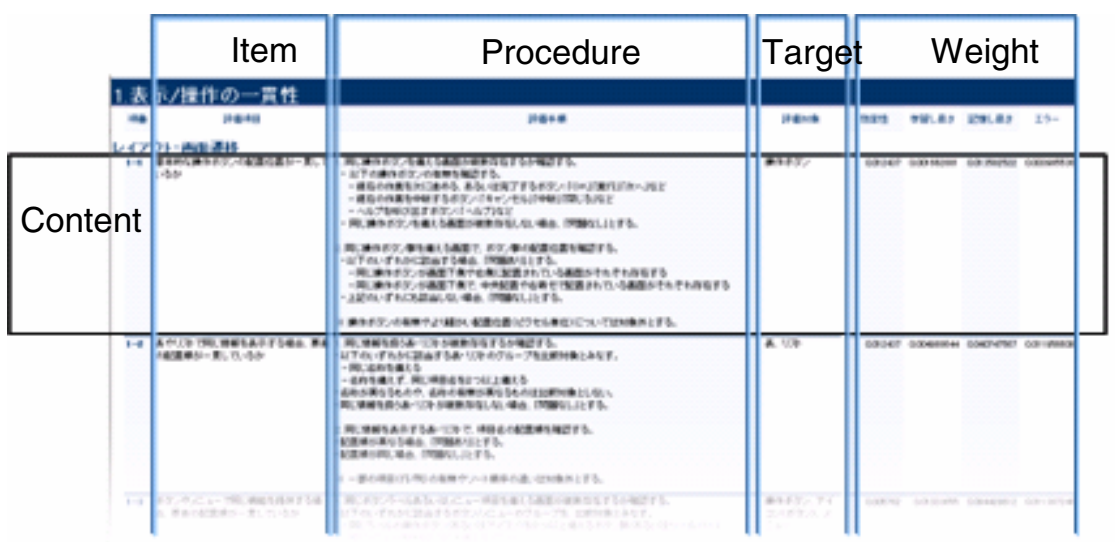

Fig. 1. Items of the checklist 
Procedure of an evaluation. This checklist consists of five sections and 84 items and is equipped with "evaluation procedure", "evaluation target", and "the weight (4 axes)" for every item (figure 1).

The flow of the evaluation was procedure-ized, and a gauge and a result in each step were described clearly to make sure that evaluation results could be judged correctly for each designated evaluation target. When a button or pull down menu was right clicked and the appropriate operation was performed, the judgment result was "No problems". When the appropriate operation was not performed, the result was "Has a problem". Additionally, in case that necessity which fits in is low which it may occur problems not to satisfy the precondition and a presence of customization, it is judged "Irrelevant". Figure 2 shows items of the checklist and a case by an illustration.

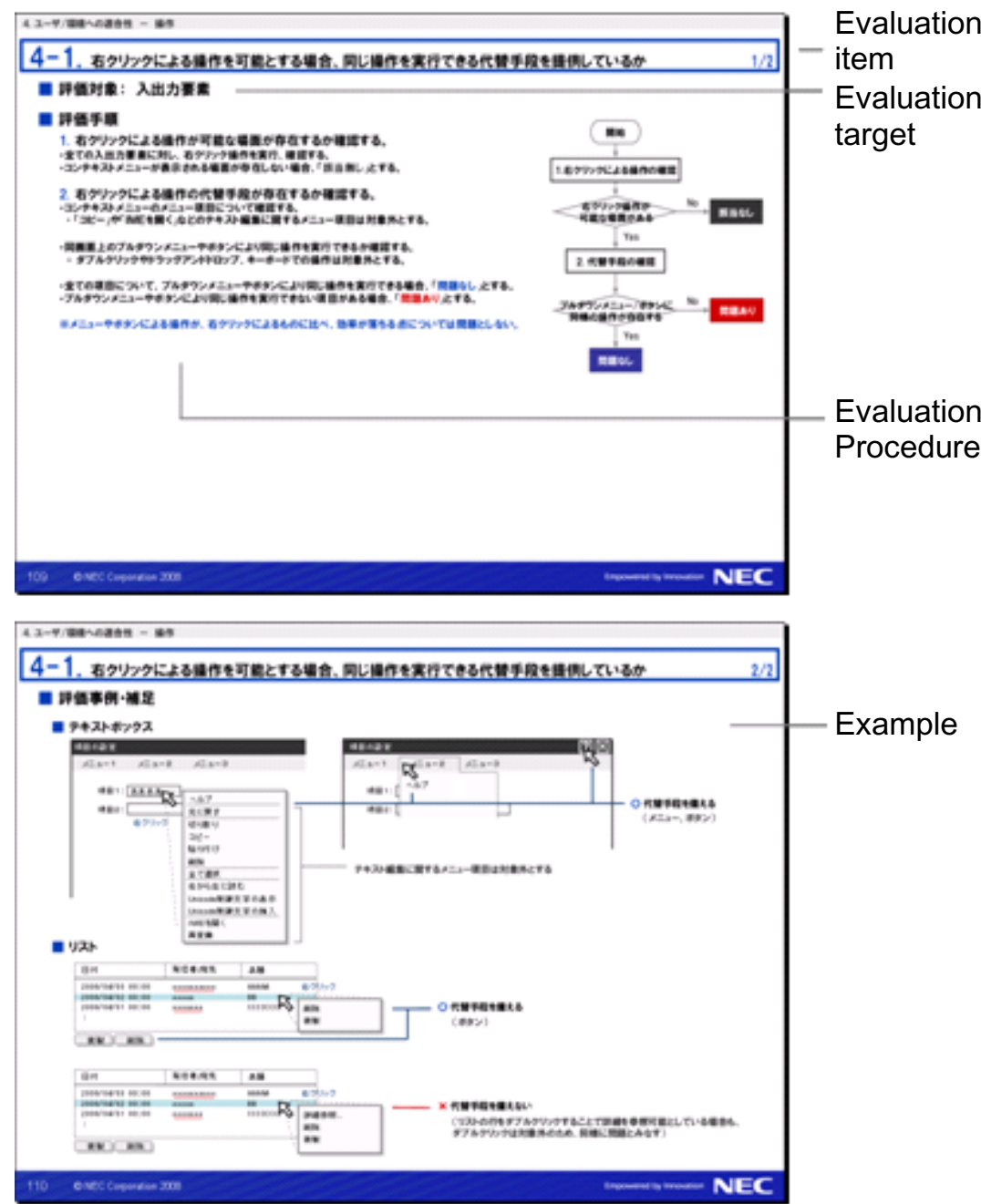

Fig. 2. Item of the checklist (details and example) 
Weight of item. To decide the weight of each item, the AHP method was applied. The feature of the AHP method is to apply paired comparison to an evaluation target according to some gauges. This method can calculate the high score of the validity compared with deciding about the weight of each element overall.

Of the five special usability qualities Nielsen advocates to writers [1], the authors chose four: "ease to learn", "errors", "ease to memorize" and "efficiency" by a gauge and decided the weight of all of these qualities in each item. The value of a paired comparison of the item was decided by conference of 3 specialists of user interface.

\section{Effectiveness Evaluation of Checklist}

\subsection{Experiment Method}

Evaluation targets. Three or five GUI windows of an e-mail software were selected as evaluation targets.

Participants (Evaluators). In total, 50 people participated in this experiment. Of these, 30 were novice college students without much experience or understand of the software's usability. On the other hand, the remaining 20 participants were expert evaluators who had experience and understanding of the software's usability. They are the researchers who are emplyed by a company.

Procedure. In accordance with the gauge described in section 2, participants evaluated some of the GUI windows prepared as evaluation targets, as explained above. By comparing novices and experts' reported results, novice participants' results can be verified to see whether it is possible to obtain results the same as those of experts.

\subsection{Experimental Result}

Each evaluation result was judged as "Has a problem", "No problems", and "Irrelevant". By comparing results, the possibility of both experts and novices obtaining the same results was tested. As an index the degree is identical between experts and novices' results, concordance rate is defined as follows [4].

The concordance rate $(\%)=$

$100 *$ (number of novices whose results agreed with those of an expert $(\%)) /($ Number of novices)

The average concordance rate obtained in this experiment was $73.75 \%$. The average concordance rates of "Has a problem", "No problems", and "Irrelevant" are show in Table 2.

Table 2. Average concordance rate

\begin{tabular}{|l|c|}
\hline Evaluation results & Concordance rate \\
\hline Has a problem & $50.6 \%$ \\
\hline No problems & $78.7 \%$ \\
\hline Irrelevant & $80.6 \%$ \\
\hline
\end{tabular}




\subsection{Discussion}

As shown in Table 2, concordance rates of "No problems" and "Irrelevant" are relatively high while that of "Has a problem" is relatively low. When problems existed, it can be said that in a lot of cases novice evaluators overlooked the problem. However, the concordance rate of $50.6 \%$ means that the rate of "more than one person of inside of $\mathrm{n}$ persons is correspondence" is $1-(1-0.506)^{\mathrm{n}}$, and big sufficiently with $94.0 \%$ at $\mathrm{n}=4$, $87.9 \%$ at $n=3$. From this, a right result is expected to be obtained when more than three people estimate a result.

\section{Practical Use of the Checklist}

This section describes the operation procedure of this checklist.

\subsection{Application of the Evaluation Items}

An evaluator judges whether an evaluation target is described as "Has a problem", "No problems", or "Irrelevant" in accordance with an evaluation procedure. The goodness of fit of the evaluation result is calculated by the weight of the judgment result and the item. Next, the methods of judging and calculating the goodness of fit of the evaluation result are described.

Judgment of a result. In the method for evaluating represented information and the consistency of operation, these items are applied to the whole screen. When they found a part of a screen with a problem by the item, evaluators judged it as "Has a problem". If there was a problem only in a part of the screen among screen groups, even when being unified during other pictures, evaluators judged it as "Has a problem" (figure 3 ). Each screen and part that was an evaluation target was evaluated in terms of the items regardless of the consistency.

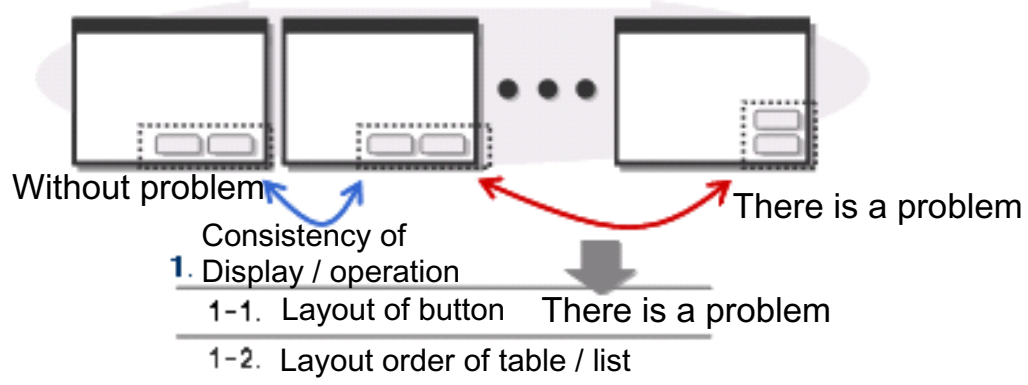

Fig. 3. Evaluation of consistency (the arrangement location of the button)

Calculation of the goodness of fit of the evaluation results. Even in the same item, there exist several evaluation targets that had different a judgment results, so goodness of fit of the evaluation results needs to be calculated by integrating the results. An 
evaluator passes a basic overall judgment by prioritizing "Has a problem", over "No problems", and then "Irrelevant". For example, if one "Has a problem" is in the results, the overall judgment is also "Has a problem". The item judged as "No problems" is weighted to check the goodness of fit of the evaluation results in accordance with the respective evaluation axes.

\subsection{Calculation of an Evaluation Result}

The amount the results concur by the same weight of each quality is the overall score on the evaluation axes. Evaluation result examples for three similar products are indicated in figure 4. Since product A clearly has the highest efficiency and product B is obviously the easiest to learn, it is possible to grasp the special quality of each product. Thus it becomes possible to confirm from four angles the evaluation result about usability by using this checklist.

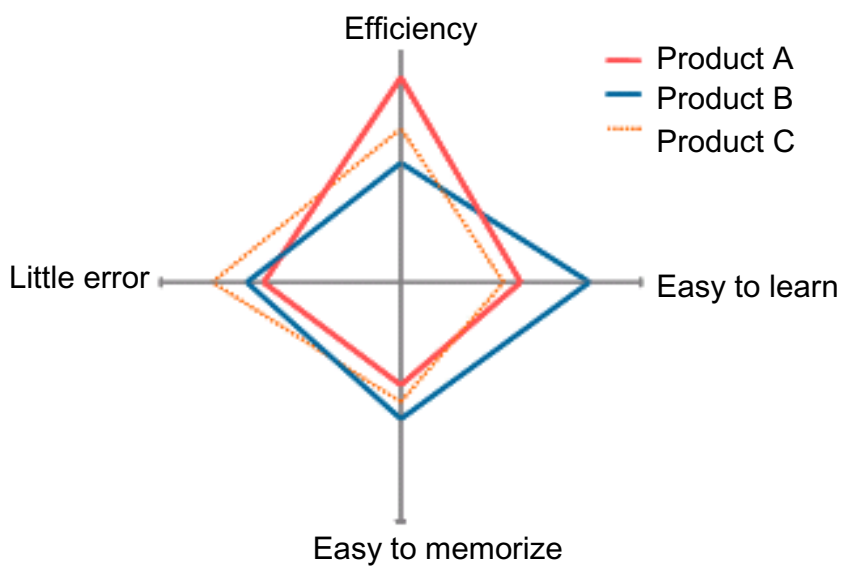

Fig. 4. Example of Evaluation Result

\section{Summary}

The authors have developed a usability quantification method in which a checklist is used that excludes blurring of results by detailing an evaluation target, an evaluation procedure, and acceptance standard. Even when an evaluator knows little about system usability, he or she can obtain objectives results on it by using this evaluation method.

\section{References}

1. Nielsen, J.: Usability Engineering. Academic Press, London (1993)

2. Ravden, S., Johnson, G.: Evaluating Usability of Human-Computer Interfaces: A Practical Method. Prentice-Hall, Englewood Cliffs (1989)

3. ISO 13407: Human-centred design processes for interactive systems (1999) 
4. Ikegami, T., Okada, H., Yoshizaka, S., Fukuzumi, S.: Proposal of usability quantification method (1) -checklist for exclusion blurring among evaluators. In: Annual conference of Information Processing Society Japan (2008) (in Japanese)

5. Okada, H., Ikegami, T., Yoshizaka, S., Fukuzumi, S.: Proposal of usability quantification method (2) -experiment for validation of a checklist. In: Annual conference of Information Processing Society Japan (2008) (in Japanese)

6. Kato, S., Horie, K., Ogawa, K., Kimura, S.: A Human Interface Design Checklist and Its Effectiveness. Transaction of Information Processing Society of Japan 36(1), 61-69 (1995) (in Japanese)

7. Ham, D.-H., Heo, J., Fossick, P., Wong, W., Park, S.-H., Song, C., Bradley, M.: Model-based approaches to quantifying the usability of mobile phones. In: Jacko, J.A. (ed.) HCI 2007. LNCS, vol. 4551, pp. 288-297. Springer, Heidelberg (2007) 